\title{
Structural Deformation of MTX Induced by Nanodrug Conjugation Dictate Intracellular Drug Transport and Drug Efficacy
}

\author{
Jun-Young Park' \\ Ja-Shil Hyun ${ }^{2}$ \\ Jun-Goo Jee ${ }^{3}$ \\ Sung Jean Park $\mathbb{D}^{2}$ \\ Dongwoo Khang (1D)
}

'Lee Gil Ya Cancer and Diabetes Institute, Gachon University, Incheon, 21999, Republic of Korea; ${ }^{2}$ College of Pharmacy and Gachon Institute of Pharmaceutical Sciences, Gachon University, Incheon, 21936, Republic of Korea; ${ }^{3}$ Research Institute of Pharmaceutical Sciences, College of Pharmacy, Kyungpook National University, Daegu, 41566, Republic of Korea; ${ }^{4}$ Department of Physiology, College of Medicine, Gachon University, Incheon, 21999, Republic of Korea
Correspondence: Dongwoo Khang Department of Physiology, College of Medicine, Gachon University, Incheon, 21999, South Korea

Tel +82-32-899-65I5

Email dkhang@gachon.ac.kr

Sung Jean Park

College of Pharmacy and Gachon Institute of Pharmaceutical Sciences, Gachon University, Incheon, 21936, Republic of Korea

Tel +82-32-899-6II3

Email psjnmr@gachon.ac.kr
Background: Understanding structural interactions between the active drug and conjugated nanoparticles is critical for optimizing intracellular drug transport and for increasing nano drug efficacy. In this regard, analyzing the conformational deformation of conjugated drugs surrounding nanoparticles is essential to understand the corresponding nanodrug efficacy.

Purpose: The objective of this study is to present an optimal synthesis method for efficient drug delivery through a clear structural analysis of nanodrugs according to the type of conjugation.

Methods and Results: In this study, the structural variation of methotrexate (MTX) surrounding carbon nanotubes, depending on the type of conjugation style, such as covalent and non-covalent (PEGylation) bonds, was investigated. Specifically, covalent bonds of MTX surrounding CNTs induced greater structural deformation compared to non-covalent bonds (ie, PEGylated CNT).

Conclusion: Greater changes in the structural variations of MTX analyzed by nuclear magnetic resonance (NMR) significantly improved the anti-inflammatory drug efficacy of human fibroblast-like synovial cells (FLS) via stable drug release in the extracellular environment and burst drug release under intracellular conditions.

Keywords: structural deformation, methotrexate, covalent conjugation, rheumatoid arthritis, carbon nanotube

\section{Introduction}

In drug therapy, the use of a minimal dose with maximum efficacy is ideal in terms of minimizing the side effects caused by drug toxicity. Nanodrug delivery systems have great advantages because they are easily taken up by target cells through activated endocytic pathways and are transported to intracellular organs without drug efflux through optimized endosomal intracellular trafficking. ${ }^{1-5}$ There are many types of nanoconjugations, such as encapsulation, direct covalent linkages (ie, disulfide bonds, amide bonds, and ester bonds), and non-covalent linkages (ie, polyethylene glycolation (PEGylation), electrostatic interaction, $\pi-\pi$ stacking, $\pi$ polar stacking, van der Waals forces, and hydrogen bonding).$^{6-11}$ However, the relationship between the structural changes of conjugated drugs and nanoparticles with respect to the type of conjugation and their influence on nanodrug efficacy has not been clearly elucidated thus far. ${ }^{12,13}$

Methotrexate (MTX) is a widely used inhibitor of dihydrofolate reductase (widely used in cancer and autoimmune disease treatment), ${ }^{14-16}$ and low-dose 
MTX is recommended as first-line pharmacotherapy for patients newly diagnosed with rheumatoid arthritis (RA). ${ }^{17,18}$ The distribution of MTX administered in low doses in the body usually occurs in compartments of extravascular tissues such as the liver, kidneys, and synovium with a known half-life of 1 hour. It has been found that $35-50 \%$ of the administered MTX binds to albumin, and the reduction in albumin concentration in the elderly has little or no effect on MTX binding. However, with widespread clinical use for the treatment of RA, the dose of MTX monotherapy has increased. ${ }^{19,20}$ Although it has excellent therapeutic efficacy, long-term administration of MTX may cause serious side effects such as bone marrow suppression, hepatitis and infection. ${ }^{21-23}$ Therefore, in order to reduce the side effects of repeated administration of high doses, it is necessary to adopt appropriate strategies to reduce the toxicity of anti-inflammatory drugs while effectively treating the symptoms of arthritis with minimal drug dose. One strategy for reducing the toxicity in the body from drug administration is to use nanomaterials as delivery systems specifically targeting cells or tissues. The types of nanocarriers used for drug delivery include carbon nanotubes (CNTs), liposomes, gold nanoparticles, polymer micelles, dendrimers, and magnetic nanoparticles. ${ }^{24}$ In particular, until now, CNTs have been mainly used for nanocarrier drug delivery system due to their advantages of being easily functionalized by surface modification through non-covalent and covalent bonds. ${ }^{25-}$ 27 The surface of CNTs can be oxidized for surface functionalization, and could coated with an amphiphilic polymer or surfactant for the efficient cellular uptake through a special endocytosis pathway. ${ }^{26,28}$ With stability by strength and excellent hardness, CNTs are widely used today as drug carriers for biomedical purposes, genetic engineering, artificial implantation, imaging, cancer treatment, antioxidant activity, and biosensing. ${ }^{25,29}$ Previous studies using MTX-CNT nano drug delivery focused on the the treatment of tumors and arthritis. ${ }^{27-29}$ However, past studies are comparative studies on the synthesis and release of nanodrugs according to CNT types, or analyzed CNT with bovine serum albumin (BSA) coating for targeting cells with controlled drug release. ${ }^{28,29}$

This study investigated the molecular structural distortion of MTX surrounding CNT through different conjugation styles and demonstrated that different binding affinity (covalent or non-covalent) can significantly influence the subsequent nano-drug efficacy.

\section{Materials and Methods}

\section{Materials Preparation}

Carboxylated and PEG-coated nanotubes (PEG-coated) were synthesized using purified nanotubes (900-1260-1G, SES Research Inc, USA) and PEG-amine (PEG $5 \mathrm{kDa}$, NOF, Japan). The synthesis of carboxylated nanotubes and PEG was carried out according to previously described. ${ }^{30}$ Methotrexate (MTX, M9929, Sigma), a chemotherapy agent and immune system inhibitor known as amethopterin, was conjugated to carboxylated and PEG-coated nanotubes.

\section{Synthesis Method and Physical Properties of Nanodrug}

The carboxylated nanotubes were sonicated (JEIOTECH. Co.) and then dispersed in 2-morpholinoethanesulfonic acid (MES) buffer (50mM, pH 6.0, M3671, Sigma) for 5 mins. Next, N-hydroxysuccinimide (NHS, 130672, Sigma) was diluted to a concentration of $400 \mathrm{mM}$ in MES buffer (pH 6.0, $50 \mathrm{mM}$ ), then added to the carboxylated nanotube solution and stirred with a vortex mixer for 5 minutes. Then, N-(3-dimethylaminopropyl)-N-ethyl carbodiimide hydrochloride (EDC, 300mM, E6383-5G, Sigma) was added to the mixture and stirred for 30 minutes. The mixture was then transferred to a filter tube (UFC910024, Ultracell ${ }^{\circledR}-100 \mathrm{~K}, 100 \mathrm{kDa}$, Millipore), centrifuged at $4000 \mathrm{rpm}$ for 15 minutes and washed at least 5 times with $50 \mathrm{mM}$ MES buffer. Next, NHS/EDCconnected nanotubes - $\mathrm{COOH}$ and MTX were mixed in a weight ratio of 1:5 in MES buffer $(\mathrm{pH}$ 6.0), and the mixture was stirred overnight at $4{ }^{\circ} \mathrm{C}$. The nanotube-MTX suspension reacted overnight was centrifuged using an Amicon filter tube (4000 rpm, Amicon YM-100K, Millipore) and filtered at least 3 times to remove the unconjugated drugs. Finally, covalently conjugated MTX on nanotubes was dispersed and stored in PBS (10010023, Gibco).

To coat the nanotubes on PEG by non-covalent bonding, the carboxylated nanotubes by acid treatment are mixed with PEG in a weight ratio of 1:5 in de-ionized water, sonicated for 30 minutes, and then filtered through the filter tube (Amicon YM-100K, Millipore) by centrifugation for $10 \mathrm{~min}$ at $4000 \mathrm{rpm}$. The PEG coated nanotubes and MTX were mixed in a weight ratio of 1:5 in MES

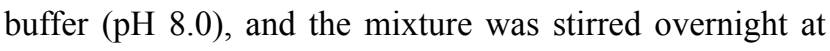
$4{ }^{\circ} \mathrm{C}$. The PEG coated nanotube-MTX (non-covalently conjugated MTX on nanotubes) suspension reacted 
overnight was centrifuged using an Amicon filter tube (4000 rpm, Amicon YM-100K, Millipore) and filtered at least 3 times to remove the unconjugated drugs. Finally, non-covalently conjugated MTX on nanotubes was dispersed and stored in PBS (10010023, Gibco). The percentage of covalent and non-covalently conjugated MTX on nanotubes was determined by measuring the weight difference between the nanodrug and PEG-coated CNTs after drying in a vacuum oven at $60{ }^{\circ} \mathrm{C}$ for at least 2 days. Drug loading was determined using the following formula: Drug loading $(\%)=$ (Weight of nanotube conjugated MTX/ Weight of oxidized nanotubes or PEG-coated nanotubes) $\times 100(\%)$. To visualize the covalent and non-covalently bonded MTX in the nanotubes, samples were placed on a copper grid and photographed using cryogenic TEM (Cryo-TEM, F20, Tecnai). All samples were plasma etched before immersion in each nano-conjugated drug solution. The samples were instantly frozen in ethane and preserved in liquid nitrogen by using a plunge freezing technique in Vitrobot (FEI).

\section{NMR Measurement}

NMR spectra were recorded on a Bruker ASCEND III 600 $\mathrm{MHz}$ spectrometer equipped with a cryoprobe. All the spectra were obtained at $298 \mathrm{~K}$. 1D ${ }^{1} \mathrm{H}$-NMR spectra of MTX and mixtures of MTX with nanotubes or PEGylated nanotubes were acquired. Furthermore, ${ }^{13} \mathrm{C}$ DEPT, ${ }^{1} \mathrm{H}-{ }^{1} \mathrm{H}$ COSY, and ${ }^{1} \mathrm{H}^{-13} \mathrm{C}$ HSQC spectra were obtained to assign all the signals of the drugs. Phase-sensitive ${ }^{1} \mathrm{H}-{ }^{1} \mathrm{H}$ 2D NOSEY spectra were obtained to monitor the drug-nanoparticle interactions and conformational states of the drugs in the complexes. 1D ${ }^{1} \mathrm{H}$ NMR spectra were obtained by solvent suppression using a watergate W5 pulse sequence with gradients. ${ }^{31}{ }^{1} \mathrm{H}-{ }^{1} \mathrm{H}$ 2D NOESY spectra were acquired at 2048 data points along t 2 dimensions; 512 free induction decays in $\mathrm{t} 1$ dimensions; number of scans, 128; relaxation delay, $5.0 \mathrm{~s}$. The mixing time for the NOESY experiments was $500 \mathrm{~ms}$. For water suppression in the NOESY spectra, a 3-9-19 pulse sequence with gradients was used. ${ }^{32}$ All spectra were processed with the Bruker Topspin 3.1 (Bruker GmbH, Germany) and Mnova 11 (Mestrelab Research). To identify the interacting region of MTX with nanoparticles such as nanotubes or PEGylated nanotubes, the chemical shift perturbation was obtained by comparing the spectra of drugs with those of drug-nanoparticle complexes. ${ }^{33}$ The changes in the chemical shifts were calculated by subtracting the proton chemical shifts of MTX-nanotube or MTX-PEGylated nanotubes complex from those of MTX. MTX spectrum was obtained at a concentration of $12 \mathrm{mg} / \mathrm{mL}$. The mixture spectrum of MTX and nanotubes was measured at a concentration of $12 \mathrm{mg} / \mathrm{mL}$ MTX and $2 \mathrm{mg} / \mathrm{mL}$ nanotube, while the mixture spectrum of MTX and PEGylated nanotubes was obtained at a concentration of $12 \mathrm{mg} / \mathrm{mL}$ MTX and $2 \mathrm{mg} / \mathrm{mL}$ PEGylated nanotubes. To estimate the structural difference between MTX in nanotubes and MTX in PEGylated nanotubes, the NOEs between the protons of MTX were analyzed. For ease of comparison, each NOE value was divided by the NOE value of the cross-peak between protons $\mathrm{H} 26$ and H26, which showed the strongest NOE value among all the NOE peaks. The resultant ratios of NOE values represent the strength of the dipolar coupling between the protons of MTX-nanotube and MTX-PEGylated nanotubes.

\section{Structure Calculation}

Structural calculations with experimental restraints were performed using the AMBER 14 package. ${ }^{34}$ The intensities of the NOE cross peaks in the NOESY spectrum are proportional to the distance between protons of less than 5 A. ${ }^{35}$ The distance restraints were subtracted from the NOE values, of which the median NOE values were set as 4.5 $\AA$. Based on these criteria, the residual NOEs were proportionally converted into distance restraints. In-house written scripts converted the manual assignments into AMBER distance restraints. The Antechamber and SQM packages of AMBER prepared AMBER-compatible parameters of MTX. The generalized Born implicit solvent model approximated the solvation effects. A restrained molecular dynamic simulation consisted of three stages: 1500-step energy minimization, 20-ps simulated annealing, and 1500-step energy minimization. The top 15 lowest energy structures of 100 structures that showed no significant violation against experimental restraints were selected as the final ensemble.

\section{Drug Release Analysis}

To assess drug release, covalently and non-covalently conjugated MTX on nanotubes were prepared in PBS ( $\mathrm{pH} 7.2$ ), PBS with 10\% FBS (pH 7.2), and ABS ( $\mathrm{pH} 5.0$ ) at an initial concentration of $1 \mathrm{mg} / \mathrm{mL}$. The nanodrugs was administered to ABS, ABS with lysozyme (1mg/mL, L1667, Sigma), and PBS, and then incubated by gently shaking for $1,2,5,10$, $24,48,72,144$ and $288 \mathrm{hrs}$ at $37^{\circ} \mathrm{C}$. At each time point, the sample was centrifuged for 5 minutes at 13,000 rpm with an ultra amicon filter (Amicon YM-100K, Millipore). The same 
amount of the supernatant obtained after centrifugation was dried at $60{ }^{\circ} \mathrm{C}$ in a vacuum oven, and the amount of MTX was analyzed by measuring the weight.

\section{Cell Isolation and Culture}

As previously described, FLS was isolated by enzymatic dispersion after obtaining synovial tissue from arthritis patients. $^{36}$ Synovial tissue samples were obtained from arthritis patients between the ages of 32 and 59 during joint surgery, and the tissues were subjected to monolayer culture. The isolation of FLS was approved by the ethics committee of Kyungpook National University for this human subject study (IRB number: 2052-040903), and prior consent was obtained from all patients. FLS were cultured in DMEM supplemented with 10\% FBS and 1\% antibiotics at $37{ }^{\circ} \mathrm{C}$, in $5 \% \mathrm{CO} 2$. FLSs were used for the experiments, at passages 3-7.

\section{EE and LE Analysis}

FLS (approximately $5 \times 10^{3}$ cells/well in 96-well plates) were treated with $500 \mathrm{ng} / \mathrm{mL}$ of covalent or non-covalent conjugation of MTX on nanotubes for the indicated 6 and $12 \mathrm{hrs}$. Next, The cells were fixed with $4 \%$ paraformaldehyde in the medium for overnight at $4{ }^{\circ} \mathrm{C}$. Permeabilization was performed at RT for $15 \mathrm{~min}$ in PBS with $0.1 \%$ Triton X-100. After blocking with $1 \%$ BSA for $2 \mathrm{~h}$ in PBS, the permeabilized cells were incubated with antibodies to the mannose 6-phosphate receptor (M6PR, a late endosome marker, ab2733, Abcam) or EEA-1 (ab2900, Abcam) at $4^{\circ} \mathrm{C}$ in the absence of light. After washing twice with PBS, the cells were incubated with either Alexa Fluor 594 goat anti-mouse IgG $(\mathrm{H}+\mathrm{L})$ (R37121, Invitrogen) or Alexa Fluor 594 goat anti-rabbit $\operatorname{IgG}(\mathrm{H}+\mathrm{L})$ (A32740, Invitrogen) for $2 \mathrm{~h}$ at RT. After washing three times with PBS, cells were mounted, and $\mathrm{EE}$ and LE intensities were visualized using confocal microscopy LSM700 (Carl Zeiss) and analyzed using the ZEN software.

\section{Real-Time Polymerase Chain Reaction (RT-PCR)}

To measure cytokine expression, real-time PCR (RT-PCR, CFX96 ${ }^{\mathrm{TM}}$ and CFX384 ${ }^{\mathrm{TM}}$, BIO-RAD) was used according to the manufacturer's manual. FLS were pretreated with MTX, PEG-coated CNTs, covalent and non-covalent conjugation of MTX on nanotubes for $2 \mathrm{~h}$, followed by stimulation with TNF- $\alpha(20 \mathrm{ng} / \mathrm{mL})$ for $12 \mathrm{~h}$. Total cellular RNA was isolated from cells (approximately $1 \times 10^{5}$ cells/well in 6 well plates), using QIAzol lysis reagent (79306, QIAGEN). First-strand complementary DNA (cDNA) was synthesized using RT Premix (Promega). The reverse transcription conditions were $45^{\circ} \mathrm{C}$ for $60 \mathrm{~min}$ and $95^{\circ} \mathrm{C}$ for $5 \mathrm{~min}$. Briefly, $9.5 \mu \mathrm{L} d \mathrm{H} 2 \mathrm{O}, 1 \mu \mathrm{L}$ sense and antisense primer solutions $(0.4$ $\mu \mathrm{M}), 2 \mu \mathrm{L}$ cDNA $(1 \mu \mathrm{g})$, and $12.5 \mu \mathrm{L}$ FastStart Universal SYBR Green Master (4913850001, Roche) were mixed to obtain a final $25 \mu \mathrm{L}$ reaction mixture in each reaction tube. PCR was performed using the following primers: TNF- $\alpha$ (forward 5-AGA GGG CCT GTA CCT CAT CT-3; reverse 5-AGA GGG CCT GTA CCT CAT CT-3), IL-1 $\beta$ (forward 5-GGA TAT GGA GCA ACA AGT GG-3; reverse 5-CCA GCT GTA GAG TGG GCT TA-3), and IL-6 (forward 5-CTT GCC TGG TGA AAA TCA TC-3; reverse 5-CTT TTT CTG CAG GAA CTG GA-3). GAPDH (forward 5-TAG ACT TCG AGC AGG AGA TG-3; reverse 5-TTG ATC TTC ATG GTG CTA GG-3) was used to verify that equal amounts of RNA were used for amplification.

\section{ELISA}

To measure the amount of TNF- $\alpha$, IL-6, and IL1b expressed by FLS, ELISA kits (Duoset DY210, DY201, DY206, and DY008, R\&D Systems, Inc. Minneapolis, MN, USA), were used according to the manufacturer's protocol. FLS were pretreated with MTX, CNTs, PEGylated CNTs, covalent MTX-CNTs, and non-covalent MTX-CNTs for $2 \mathrm{hrs,} \mathrm{fol-}$ lowed by stimulation with TNF- $\alpha(20 \mathrm{ng} / \mathrm{mL})$ for $12 \mathrm{hrs}$. After washing with PBS and culturing for $12 \mathrm{hrs}$, the conditioned media was collected. Levels of TNF- $\alpha$, IL-6, and IL1b were determined by ELISA. After applying stop solution, absorbance was measured at $450 \mathrm{~nm}$, and wavelength was corrected at $570 \mathrm{~nm}$ by using a microplate reader (Asys UVM340, Biochrom Ltd, Cambridge, UK) following the manufacturer's protocol.

\section{Ethics}

Fibroblast-like synoviocytes (FLS) were isolated from 59 to 68 years old patients suffering from rheumatoid arthritis in Kyungpook National University. All procedures for cell isolation were carried out after obtaining written consent from all patients in accordance with the Declaration of Helsinki and approval by the Institutional Ethics Committee (IRB number: 2052-040903).

\section{Statistical Analysis}

Analysis of statistical significance was analyzed with Analysis of Variance (ANOVA) for three or more samples followed by a Tukey multiple comparison test. Statistical 
significance of the $\mathrm{p}$-value was determined as $* \mathrm{p}<0.05$, $* * \mathrm{p}<0.01$, and $* * * \mathrm{p}<0.001$.

\section{Results}

\section{Structural Analysis of MTX by Nano Conjugation Styles}

A schematic illustration shows covalently conjugated MTX and non-covalent (PEGylation) conjugated MTX on nanotubes (Figure 1A). Interestingly, the transmission electron microscopy (TEM) analysis revealed that in covalently conjugated MTX, the contact angles on the surface of nanotubes were small compared to those of noncovalently conjugated MTX (Figure 1B). This indicates that the covalently conjugated MTX molecules adhered more strongly to nanotubes than non-covalently conjugated MTX.). Energy dispersive X-ray spectroscopy (EDS) analysis clearly showed the signals of $\mathrm{C}$ (carbon: CNT and PEG), $\mathrm{O}$ (oxygen: $\mathrm{CNT}$ and PEG) and $\mathrm{N}$ (nitrogen: PEG) at the same position (Figure S1). In addition, based on the nuclear Overhauser effect (NOE) data that reflects the distances between atoms, the structure of MTX conjugated on nanotubes (both covalent and noncovalent) was constructed using an assisted model building with an energy refinement (AMBER) force field. ${ }^{34}$ The root mean square deviations (RMSD) of heavy atoms in an ensemble (100 conformers) was $0.013 \AA$ in both cases, indicating a high precision of measurement (Table S1). Interestingly, the structural comparison between covalent and non-covalent conjugation on nanotubes revealed dissimilarity in the overall conformation (Figure 1C-F). The intrinsic structure of MTX should be very flexible and freely rotatable, which may be supported by the absence of measurable NOEs in the Nuclear Overhauser effect spectroscopy (NOESY) spectrum of MTX (Figure S2). However, the structures of MTX on nanotubes were more ordered and restricted because strong NOEs between protons of MTX was found in the NOESY spectra (Table $\underline{\mathrm{S} 2}$ and Figure S2). Conformational differences were distinctly observed around ring $\mathrm{C}$, which links ring $\mathrm{AB}$ and the carboxylic groups of MTX. In the case of covalent conjugation on nanotubes, ring $\mathrm{C}$ faces $\mathrm{H} 6$ and ring $\mathrm{AB}$ (Figure 1C). The angle between plane 1 of rings $\mathrm{A}$ and $\mathrm{B}$ and plane 2 of ring $\mathrm{C}$ was approximately $63^{\circ}$. The carboxylic group located at the terminal of MTX also stretched towards ring AB of MTX (Figure 1C). However, this conformational characteristic was altered in the non-covalent conjugation on the nanotubes. The angle between plane 1 of rings $\mathrm{A}$ and $\mathrm{B}$ and plane 2 of ring $\mathrm{C}$ increased to $122^{\circ}$. The orientation of the carboxyl group was not identical to that of the covalent MTX-CNTs (Figure 1E). This result may imply that the induced conformations in both nanotubes have different characteristics, which may be caused by the altered hydrophobic contacts between the ring protons of MTX (Figure 1E). It is very probable that the hydrophobic environment formed by PEG additionally influenced the content of protons of the nanotubes. The different distances between several protons are shown in Figure $1 \mathrm{~F}$. For example, the average distance between $\mathrm{H} 6$ on ring $\mathrm{B}$ and $\mathrm{H} 18 / 20$ on ring $\mathrm{C}$ was close to $5 \AA$ in the non-covalently conjugated MTX on nanotubes, whereas the average distance was shorter in the MTX covalently conjugated on nanotubes (Figure 1C-F). In conclusion, the obtained NOESY analysis clearly shows greater conformational changes (small distance of proton pairs) in molecular structures of covalently conjugated MTX on nanotubes than non-covalently (PEGylated) conjugated MTX (larger distance of proton pairs) on nanotubes.

\section{Characterization of Covalent and Non-Covalent Conjugation of MTX on Nanotubes}

The material properties of two different structural variations of MTX on nanotubes were analyzed using particle size analysis, electrokinetic potential, ultraviolet-visible (UV-vis), and Fourier transform infrared spectroscopy (FTIR) (Figure 2A-D). The average length and size of free MTX, free nanotubes, covalent, and non-covalent conjugation of MTX on nanotubes (PEGylated) were 99, 166, 173, and $213 \mathrm{~nm}$, respectively (Figure 2A). The polydispersity index (PDI) was less than 0.3 , for all tested samples. The electric potential changes due to the surface charge of the samples revealed that free MTX, free nanotubes, PEG-coated nanotubes, covalent, and non-covalent conjugation of MTX on nanotubes were all negatively charged (Figure 2B). In addition, UV-vis spectroscopy showed absorbance peaks at 258, 303, and $372 \mathrm{~nm}$ for MTX (Figure 2C). The MTX peak on MTX-CNT was redshifted by approximately $10 \mathrm{~nm}$, while the MTX peaks on MTX-PEG-CNT were not different from those of free MTX (Figure 2C). The red shift of the drug absorbance peak represents strong evidence of covalently conjugated MTX on nanotubes. ${ }^{37}$ The amount of loaded MTX on covalently and non-covalently conjugated MTX on 
A

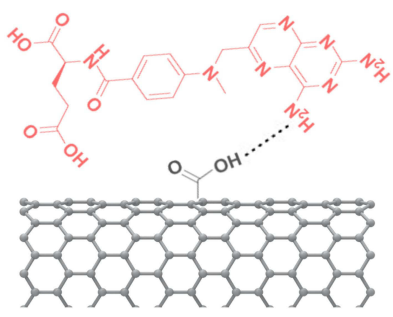

Covalent MTX-CNT

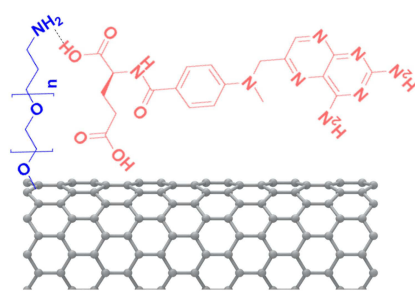

Non-covalent MTX-CNT
B
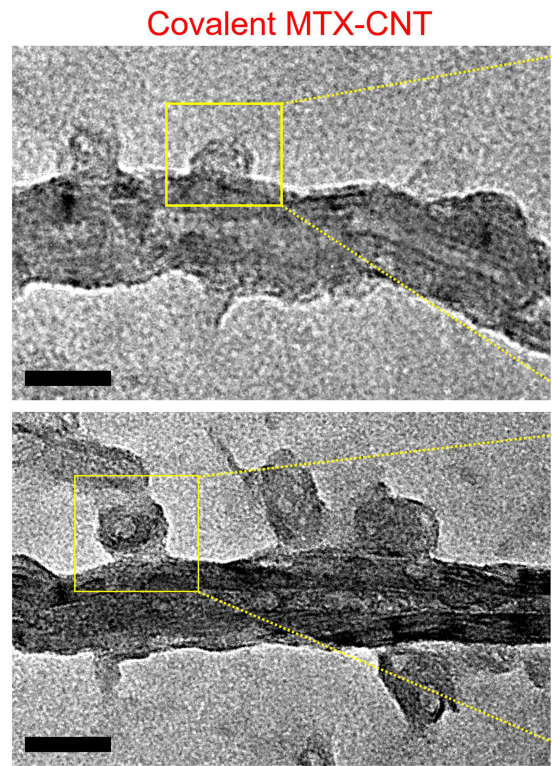

Non-covalent MTX-CNT
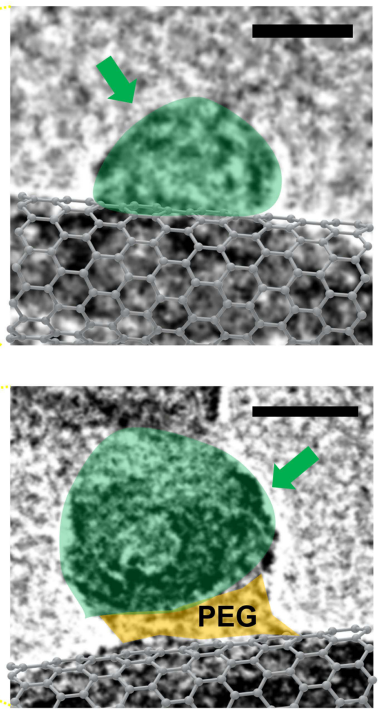

c Covalent MTX-CNT

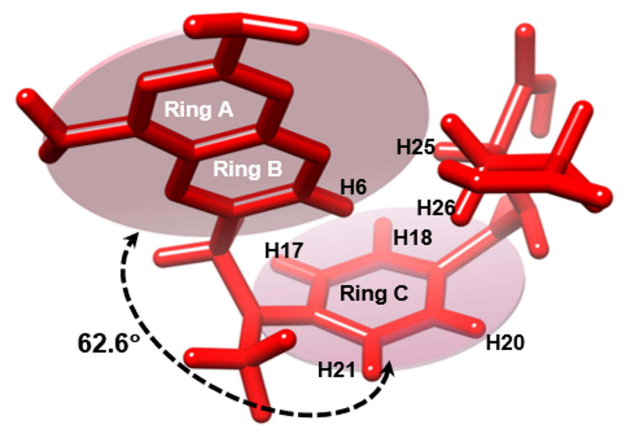

Non-covalent

E MTX-CNT

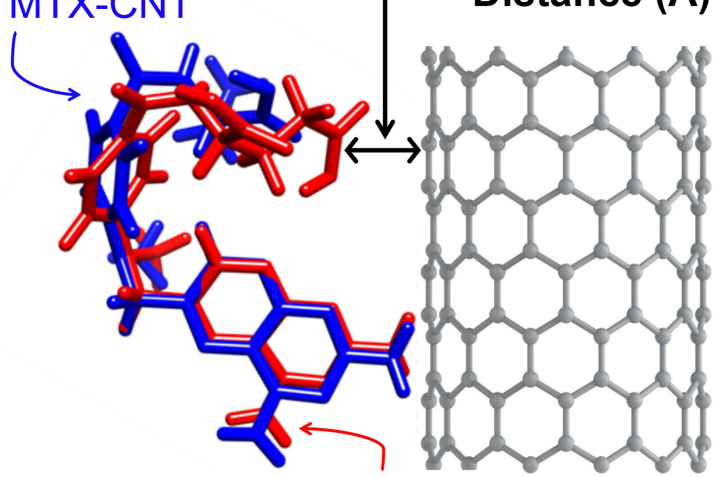

Covalent MTX-CNT

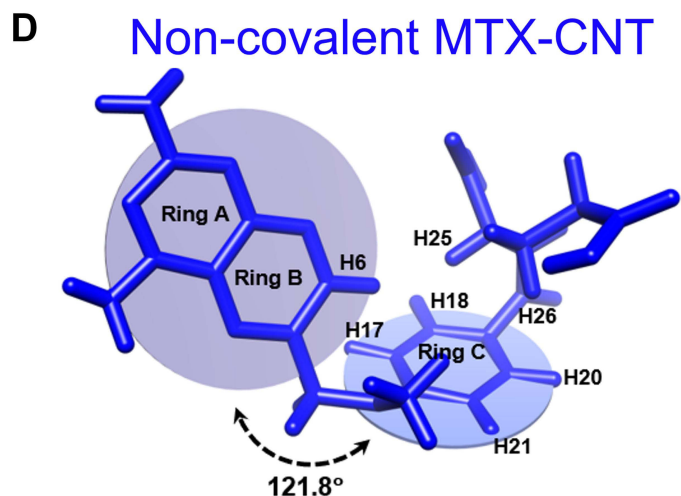

$\mathbf{F}$

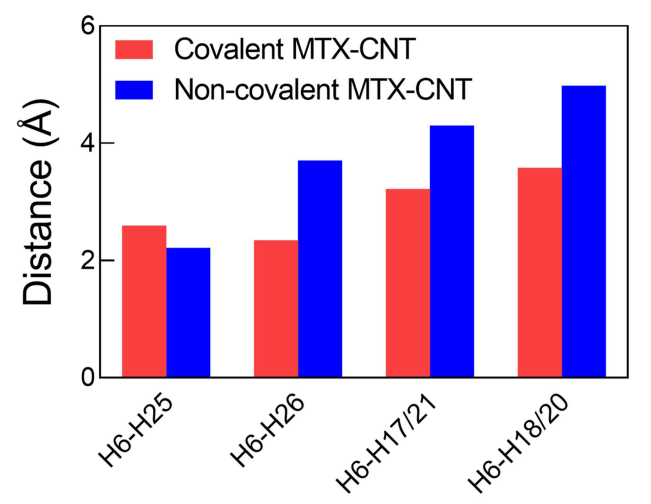

Proton Pairs

Figure I Structural change of nanodrugs. (A) Corresponding chemical bonding illustrations of covalent methotrexate (MTX)-carbon nano tube (CNT) and non-covalent MTXCNT. (B) Transmission electron microscopic (TEM) images of covalent and non-covalent MTX-CNT, and the scale bar is $20 \mathrm{~nm}$. (C-D) Image shows the structural differences in chemical bonds between covalently and non-covalently conjugated nanodrugs. The structures were calculated on the basis of nuclear Overhauser effect (NOE) information as described. One hundred conformers showing lowest amber energy values were obtained for each complex. The representative MTX structures with the lowest energy are shown: (C) covalent MTX-CNT and (D) non-covalent MTX-CNT. The angles between plane I of ring A and B and plane 2 of ring C are depicted with arrows. Several protons which showed different NOE values in both structures are depicted as well. (E) Superimposed structures between MTX attached on nanotubes (red) and PEGylated CNT (blue). The manual alignment was performed based on the orientation of the ring AB. (F) The representative distances that showed large difference between the covalent MTXCNT (red bars) and the non-covalent MTX-CNT (blue bars) are shown in the bar chart. The distances of each pair was calculated from.Table S2 

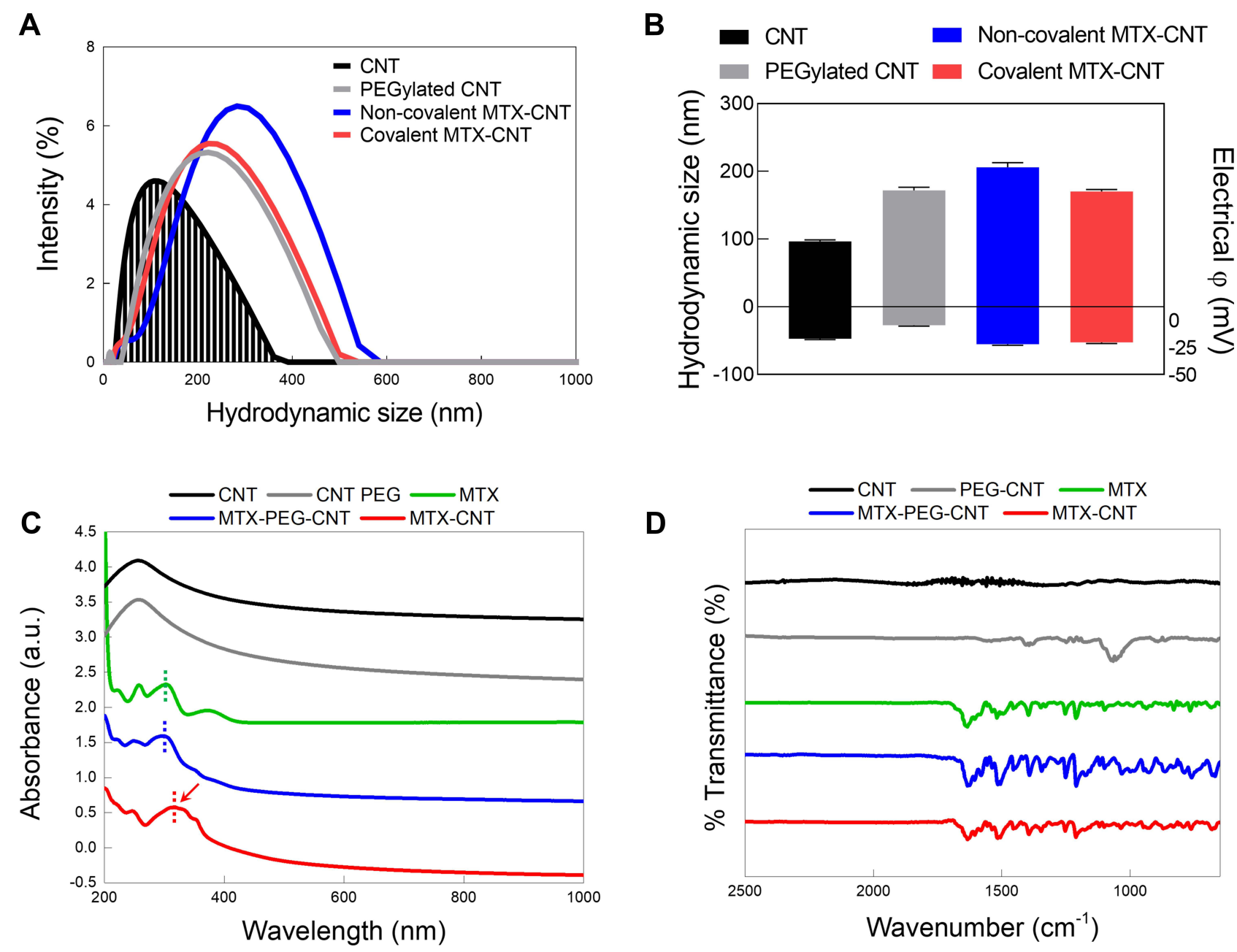

Figure 2 Physicochemical properties of nanodrugs. (A) Hydrodynamic size analysis of methotrexate (MTX), carbon nanotube (CNT), polyethylene glycolated (PEGylated) CNT, non-covalent MTX-CNT, and covalent MTX-CNT. (B) Zeta potential analysis of MTX, CNT, PEGylated CNT, non-covalent, and covalent MTX-CNT. An increase in the average size of a drug bound to CNT and a negative charge on the drug surface indicates stable drug binding. (C) Ultraviolet-visible spectra (UV-vis) of MTX, noncovalent MTX-CNT, and covalent MTX-CNT showing identical peaks at $252 \mathrm{~nm}$. (D) Fourier transform infrared spectroscopy (FTIR) peaks of MTX, CNT, PEGylated CNT, non-covalent, and covalent MTX-CNT. Identical MTX peaks were observed in MTX, non-covalent, and covalent MTX-CNT.

nanotubes was quantified by analyzing the absorption spectrum by using standard curves (Figure S3a-b). The differences in absorbance peaks between MTX and the base nanotubes at a specific wavelength $(303 \mathrm{~nm})$ corresponded to the amount of loaded MTX drug (Figure 2C). The chemical changes that could be induced by covalent and non-covalent conjugated MTX on nanotubes were confirmed by FTIR spectroscopy. FTIR revealed agreement of major MTX IR peaks between the free MTX drug and the covalent or non-covalently conjugated nano drug (Figure 2D). In addition, the chemical shift perturbation of MTX according to the covalent or non-covalent conjugation was monitored by the ${ }^{1} \mathrm{H}-\mathrm{NMR}$ spectrum, of which result is summarized in the first section of Supplementary materials. Briefly, the ${ }^{1} \mathrm{H}-\mathrm{NMR}$ spectrum of MTX was obtained (Figure S4) and proton chemical shifts of the MTX were assigned (Table S3). This MTX spectrum was compared to the spectrum of MTX-CNT (Figure S5a) and to the spectrum of MTX-PEG-CNT (Figure S5b). Resultantly, it was found that the chemical shifts of MTX were differently moved depending on the type of conjugation (Figure S5c).

\section{MTX Release Analysis}

Dynamic drug release analysis depending on the structural variation of MTX by covalent and non-covalent conjugation was monitored under various physiological conditions, such as pH 5.0 and 7.0 (Figure 3A-B). To mimic intra- and extra-cellular blood environments, PBS, acetate-buffered saline (ABS, acidic condition), and fetal 

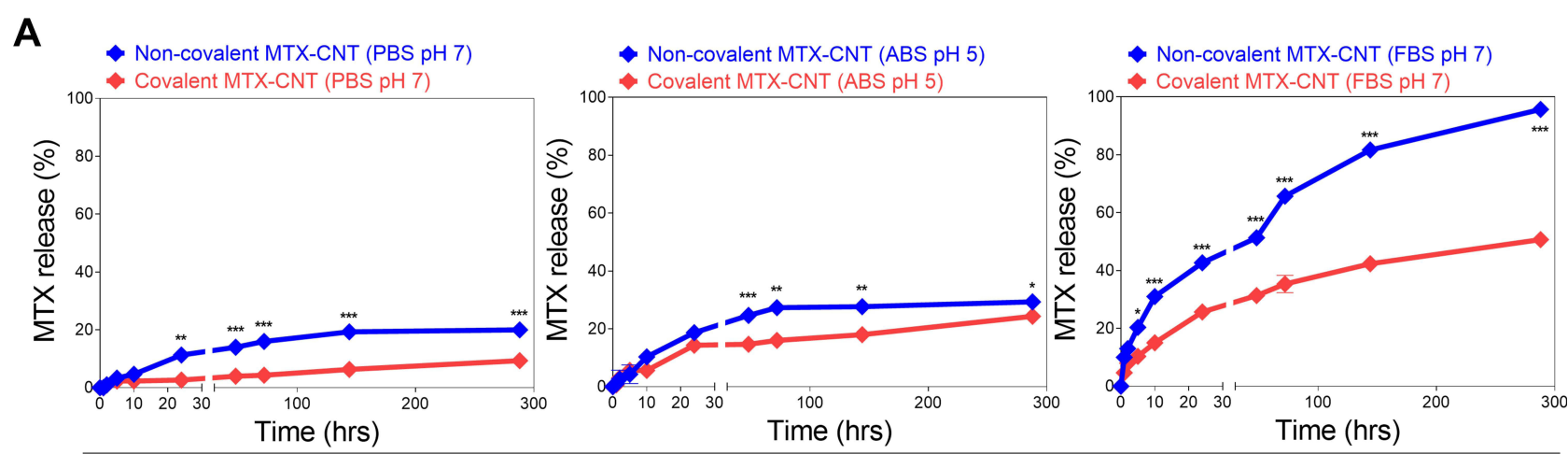

Extra-cellular

B
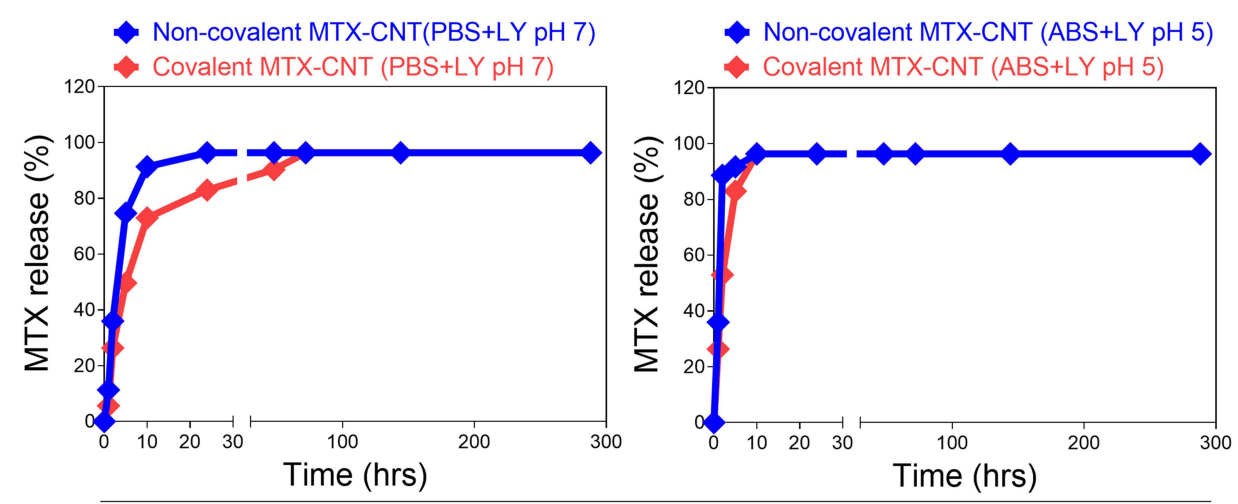

Intra-cellular

C

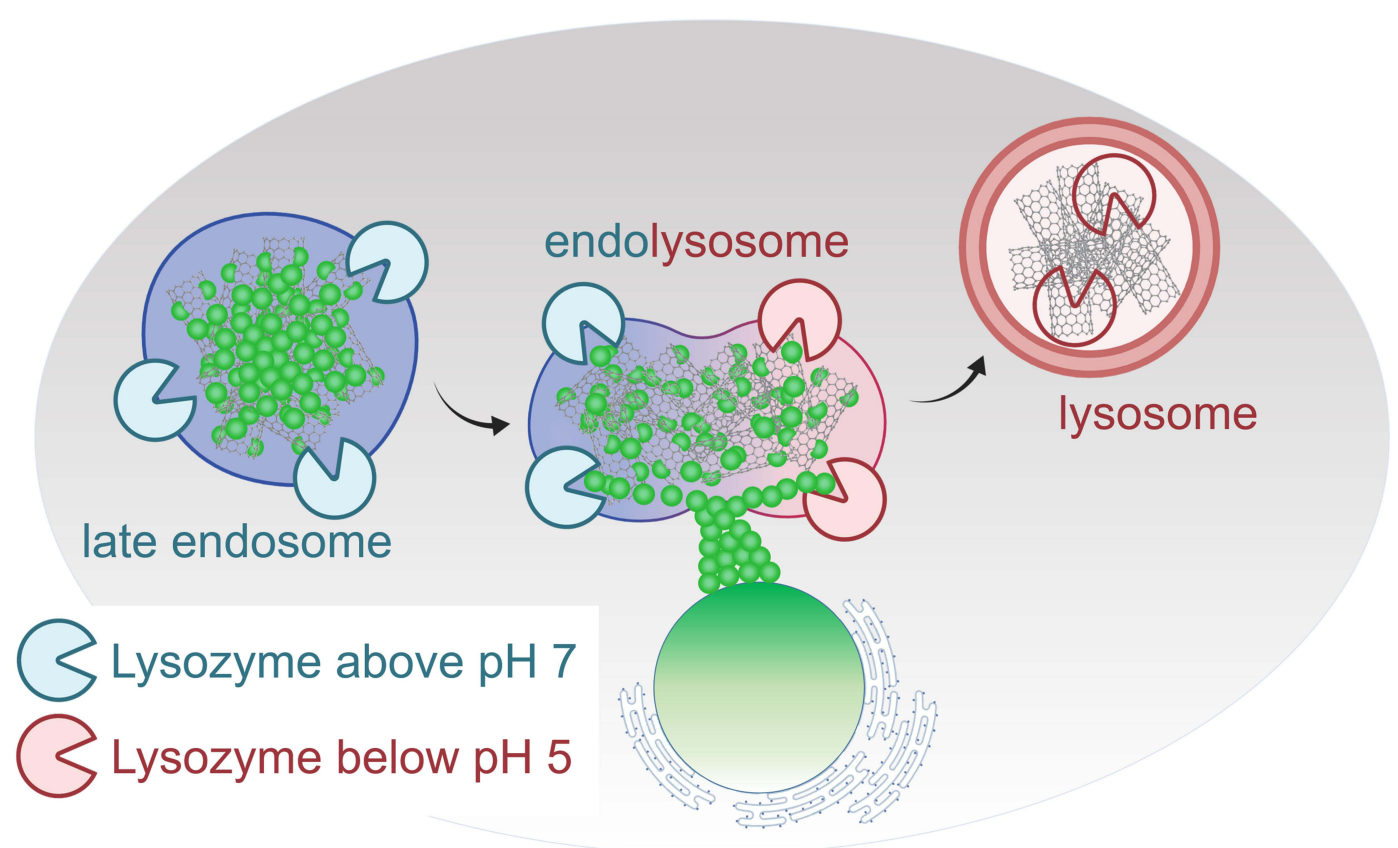

Figure 3 Drug release analysis. (A) Methotrexate (MTX) release from non-covalent MTX-carbon nanotube (CNT) and covalent MTX-CNT were analyzed in neutral condition (ie, PBS), acidic condition (ie, acetate-buffered saline (ABS)) and Fetal bovine serum (FBS) condition (ie, pH 7), (B) lysozyme-supplemented condition (ie, pH 7), and acidic lysozyme-supplemented condition (ie, $\mathrm{pH} \mathrm{5)}$ up to $288 \mathrm{hrs}$. (C) Schematic illustration of late endosome-lysosome delivery with burst drug release in the endolysosome stage. Covalently bound nanodrugs maintain stable binding compared to non-covalently bound nanodrugs in extra-cellular environments, and show burst drug release in intra-cellular environments. All data represent the mean \pm SEM $(n=3) . * p<0.05, *_{p}<0.01$ and $* * * p<0.001$. 
bovine serum (FBS supplemented with blood enzymes) were used. In addition, all nanodrugs used for MTX release analysis were performed under the same concentration condition of $1 \mathrm{mg} / \mathrm{mL}$ based on MTX. Under physiological conditions, MTX release from PEGlyated nanotubes was faster than that from covalently conjugated nanotubes (Figure 3A-B). This suggests weak interactions between MTX and PEGylated nanotubes compared to the strong interactions between covalent MTX and nanotubes resulted in rapid drug release. The drug release level of covalently conjugated MTX on nanotubes was below $10 \%$ in the PBS condition; however, MTX release from non-covalent conjugated nanotube was $20 \%$ after $288 \mathrm{~h}$ (Figure 3A). In the blood mimic condition (FBS of $10 \%$ ), covalently conjugated MTX on nanotubes was maintained at approximately $40 \%$, while non-covalently conjugated MTX on nanotubes showed complete release of MTX after 288 h (Figure 3A). Thus, PEGylated MTX was completely released from the nanotubes after $288 \mathrm{~h}$. Under acidic conditions (ABS), the drug release of non-covalently and covalently conjugated MTX on nanotubes was maintained below $30 \%$ (Figure $3 \mathrm{~A}$ ). The $\mathrm{pH}$ level of the synovial fluid of arthritis joints under inflammatory conditions may be weakly acidic around $\mathrm{pH} 6$, and thus, the obtained results indicate that the release of MTX was a slow, stable release of covalently and non-covalently conjugated MTX on nanotubes at $\mathrm{pH} 5$ in inflammatory pathophysiological environments. This is a favorable condition because conjugated MTX should be stable on nanotubes before cell entry. In contrast, plasma proteins highly influenced the release of MTX from the nanotubes. FBS contains various enzymes, which may facilitate the release of MTX by breaking the bonds on nanotubes. $^{38,39}$ It should be noted that the stability of covalently conjugated MTX on nanotubes is greater than that of the non-covalently conjugated MTX on nanotubes in FBS media (Figure 3A). Furthermore, when treated with high level of lysozymes (intra-cellular condition), drug release analysis showed the burst release of MTX on nanotubes, mediated by lysozymes (at both $\mathrm{pH}$ 7.0 and 5.0) (Figure 3B). A large amount of lysozyme exists inside late endosomes and lysosomes, and thus, MTX is quickly released from the nanotube regardless of the conjugation. Thus, covalent conjugations are more stable than non-covalent conjugations in extra-cellular conditions, but they are highly responsive to acidic hydrolases (Figure 3B).

\section{FLS Uptake Analysis}

The intracellular uptake pathways of covalently and noncovalently conjugated MTX on nanotubes in fibroblast-like synovial cells (FLSs) were analyzed. Streptavidin-Alexa 488 was co-conjugated on covalently and non-covalently conjugated MTX on nanotubes $(\lambda \mathrm{ex}=485 \mathrm{~nm}$ and $\lambda \mathrm{em}=$ $535 \mathrm{~nm}$ ) and were examined using confocal analysis (Figure 4A-D). It was clearly demonstrated that the TNF- $\alpha$ stimulated FLS cultured with covalently and non-covalently conjugated MTX-Alexa 488 on nanotubes exhibited increased uptake intensity compared to non-TNF- $\alpha$ treated samples (after $6 \mathrm{~h}$ of incubation) (Figure $4 \mathrm{~A}$ and $\mathrm{C}$ ). Importantly, regardless of TNF- $\alpha$ stimulation, the uptake intensity of the covalently conjugated MTX on nanotubes was greater than that of non-covalently conjugated MTX on nanotubes (Figure 4A-B). In addition, to identify the uptake pathways of covalently and non-covalently conjugated MTX on nanotubes, caveola-mediated endocytosis inhibitors (genistein, GEN), micropinocytosis (5- (N-ethyl cells were treated with inhibitors of -N-isopropyl) amiloride (EIPA), and clathrin-mediated endocytosis (chlorpromazine, CPZ) were used (Figure 4B). Specifically, the uptake pathway of covalent MTX-nanotubes was strongly dependent on caveolar (81\%)-mediated endocytosis (Figure 4B and D). On the other hand, clathrin (47\%) intracellular uptake pathways were significantly increased by noncovalent conjugation of MTX on nanotubes (Figure 4B and D). As such, covalent conjugation strongly induced caveolin uptake, whereas non-covalent conjugation significantly induced clathrin uptake pathways in FLS. Considering the size and electric potential analysis (Figure 2B), it was speculated that altered uptake pathways are influenced by nanoconjugation styles (covalent and noncovalent), and the obtained results are also identical to those of previous studies. ${ }^{30}$

\section{Intra-Cellular Trafficking (EE and LE Analysis)}

Confocal analysis showed greater early endosome (EE) intensities for both covalently and non-covalently conjugated MTX on nanotubes after 6 and $12 \mathrm{~h}$ (Figure 5A). In contrast, late endosome (LE) intensity was only observed in covalent conjugation of MTX on nanotubes, whereas noncovalent conjugation of MTX on nanotubes did not show any LE after $12 \mathrm{~h}$ (Figure 5B). This means that the formation of LE vesicles is only possible by covalent conjugation, and the obtained result is in agreement with a previous study. ${ }^{30}$ 
A

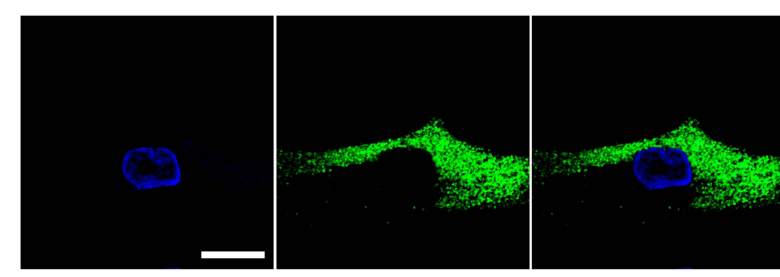

Non-covalent MTX-CNT

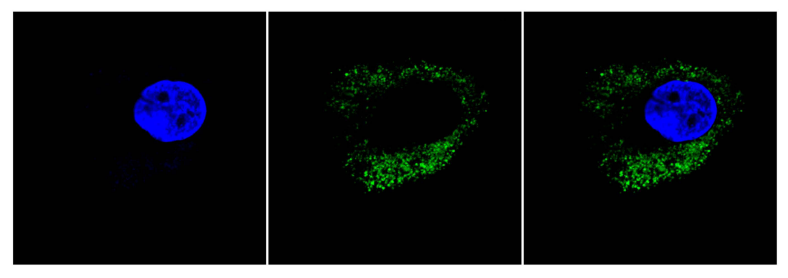

+ TNFa stimulation

B
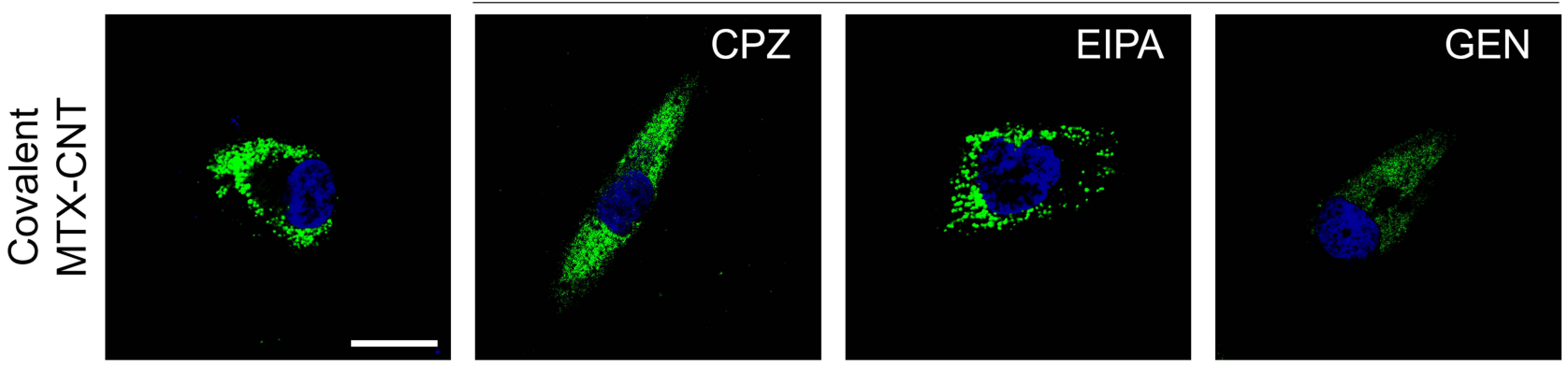

+ TNF- $\alpha$
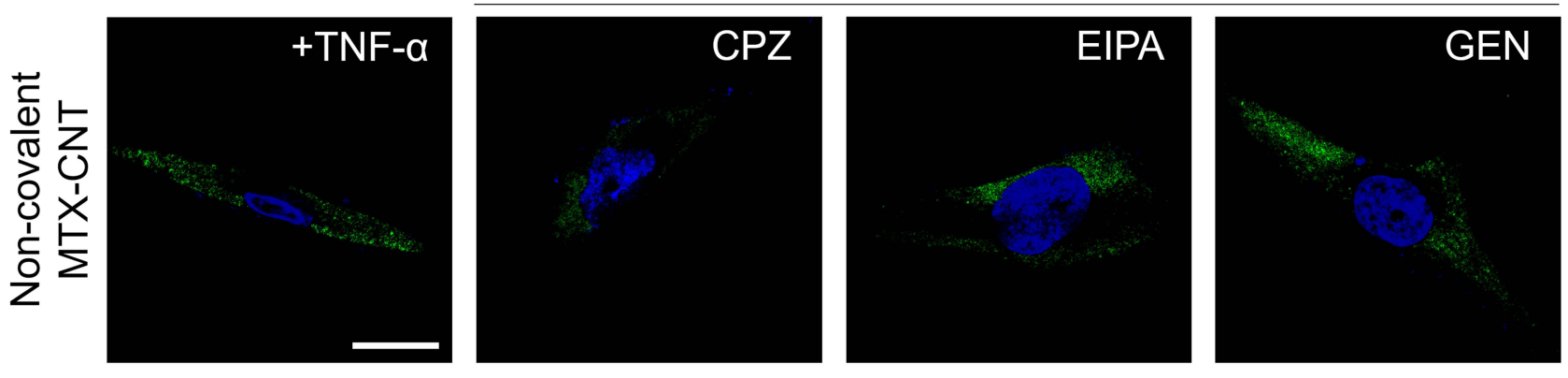

C

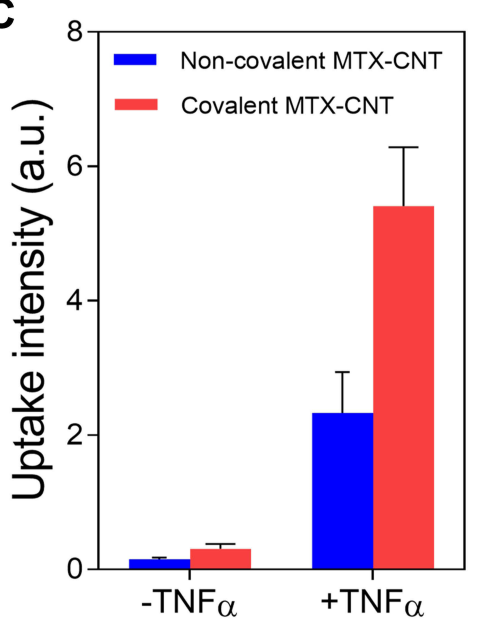

D

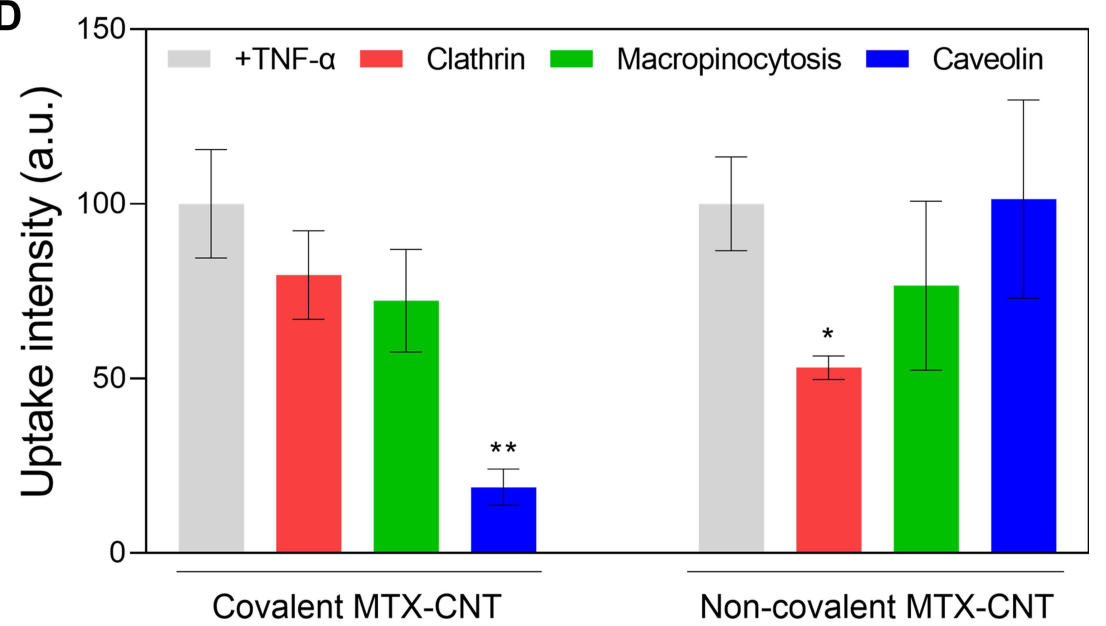

Figure 4 Human fibroblast-like synovial cell (FLS) uptake analysis. (A) Confocal images shows free FLSs and TNF- $\alpha$-stimulated FLSs treated with the covalent methotrexate (MTX)-carbon nanotube (CNT) and non-covalent MTX-CNT. In FLS stimulated with TNF-a, increased uptake of nanodrugs was confirmed, and the uptake of covalent MTXCNT was increased in FLS than that of non-covalent MTX-CNT. The scale bar is $20 \mu \mathrm{m}$. (B) Confocal image visualizing TNF- $\alpha$-stimulated FLS treated with nano-drug. TNF$\alpha$-stimulated FLS treated with different types of absorption inhibitors were visualized and analyzed. The scale bar is $20 \mu \mathrm{m}$. It was shown that the uptake of covalently conjugated MTX on nanotubes FLS occurred using the caveolar-mediated endocytosis pathway, whereas the non-covalent conjugation of MTX on nanotubes was dependent on the clathrin-mediated endocytosis pathway. (C) Fluorescence intensity (uptake) analysis of free FLSs and TNF- $\alpha$-stimulated FLSs treated with the nanodrug. (D) Fluorescence intensity analysis of TNF- $\alpha$-stimulated FLS for comparison of major intracellular uptake pathways between covalent and non-covalently bound drugs. All data represent the mean $\pm \operatorname{SEM}(\mathrm{n}=3)$. $*_{p}<0.05$ and $* *_{p}<0.01$. 

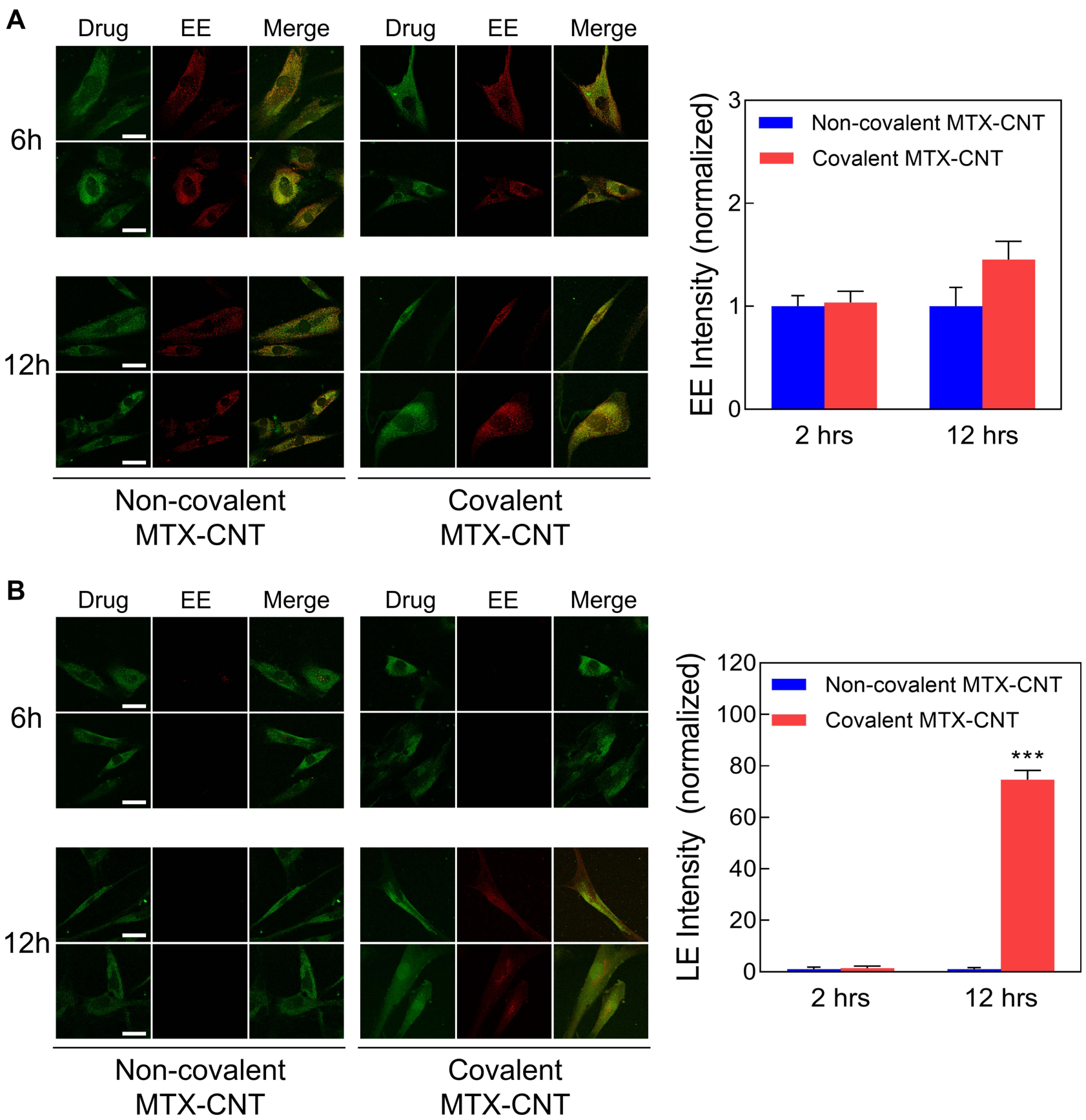

Figure 5 Intracellular trafficking. (A) Confocal microscopic analysis of early endosome (red) and covalent methotrexate (MTX)-carbon nanotube (CNT) (both covalent and non-covalent conjugation) for the desired time points (6 and I2 h). The scale bar is $20 \mu \mathrm{m}$. The normalized intensities of early endosomes after the treatments of covalent and non-covalent MTX-CNT at a certain time point ( 6 and $12 \mathrm{~h}$ ) were quantified through calculation of fluorescence intensities of confocal images. (B) Confocal microscopic analysis of late endosome (red) and covalent MTX-CNT (green) complexes after the incubations with covalent or non-covalent MTX-CNT at indicated time points (6 and 12 h). The scale bar is $20 \mu \mathrm{m}$. The fluorescence intensities of late endosomes after incubating with covalent MTX-CNT and non-covalent MTX-CNT for 6 and I2 h were calculated by normalizing the fluorescence intensities of confocal images. All data represent the mean $\pm S E M(n=3)$. $* * * p<0.00 \mathrm{I}$.

The obtained LE results showed that most PEGylation coatings did not develop into the LE from the EE stage due to the rapid release of the drug, whereas covalent conjugation of MTX on nanotubes, which are stable bonds formed during the early late endosome stage, sustained LE stages. In conclusion, the intra-cellular trafficking analysis clearly indicates that covalent conjugation shows a more stable drug conjugation style in terms of intracellular drug delivery system: sustained nanodrug formation before the lysosome stage without release into the cytosol (Figure 5A-B). ${ }^{40}$ 
A

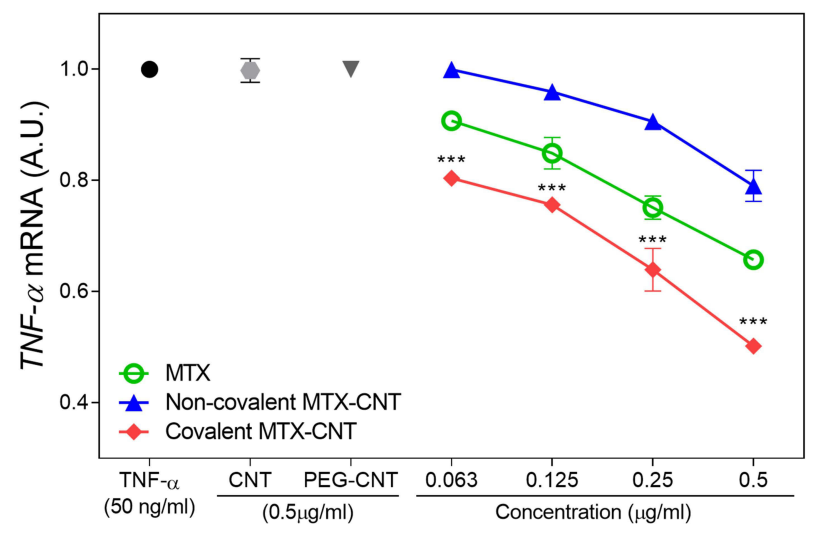

C

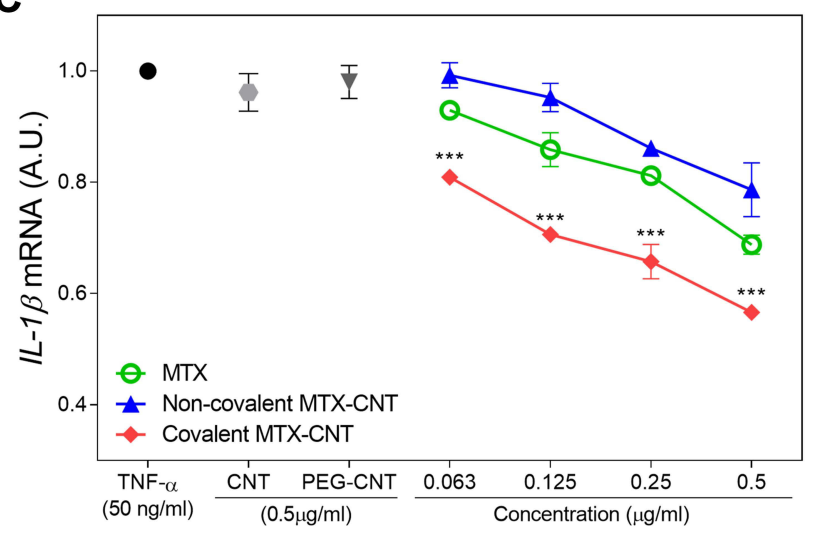

E

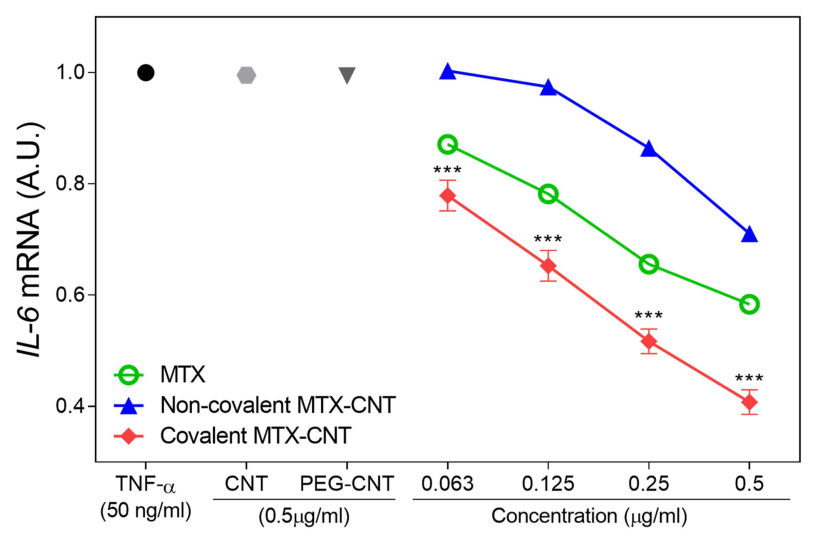

B
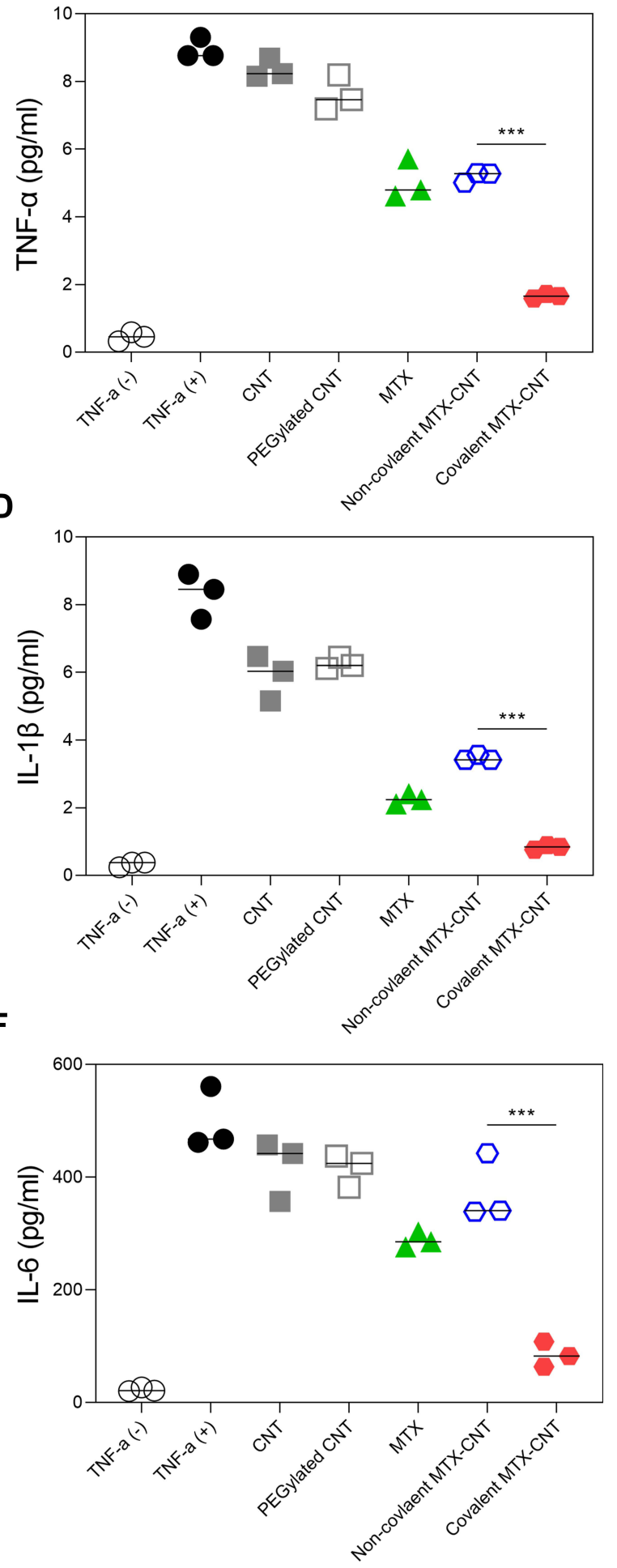

Figure 6 Anti-inflammatory efficacy. Low-dose therapeutic efficacy of covalent methotrexate (MTX)-carbon nanotube (CNT) compared to non-covalent MTX-CNT and free MTX in TNF- $\alpha$-stimulated human fibroblast-like synovial cells (FLS) Suppression of (A) TNF- $\alpha$, (C) IL-I- $\beta$, and (E) IL-6 mRNA levels in TNF- $\alpha$-stimulated FLS. The data show that covalently conjugated MTX on nanotubes treatment led to a greater suppression of inflammatory cytokines than non-covalently conjugated MTX on nanotubes in FLSs. Suppression of protein levels was detected by ELISA in TNF- $\alpha$-stimulated FLSs after treatment with nanodrugs for 24 h. (B) TNF- $\alpha$, (D) IL-I- $\beta$, and (F) IL-6 protein levels were significantly suppressed by covalent MTX-CNTs compared to polyethylene glycolated (PEGylated) MTX-CNTs. All data are presented as mean \pm SEM ( $\mathrm{n}=3$ ). $* * * p<0.001$. 


\section{Anti-Inflammatory Response}

The expression of major inflammatory markers (mRNA and protein level) was analyzed to examine the antiinflammatory response of nanodrugs on FLS (Figure 6AF). FLS cells were pre-incubated with free MTX, covalently, and non-covalently conjugated MTX, separately, on nanotubes and stimulated with TNF- $\alpha$ for $12 \mathrm{~h}$. Then, the mRNA expression of TNF- $\alpha$, IL-1 $\beta$, and IL- 6 genes was monitored. Covalent MTX significantly reduced the mRNA levels of major pro-inflammatory cytokines, such as TNF- $\alpha$, IL-1 $\beta$, and IL-6, compared to free MTX and non-covalent (PEGylated) MTX on nanotubes, which implies a lower dose suppression of the inflammatory response by covalent conjugation of MTX on nanotubes (Figure 6A, C, and E). The obtained mRNA results led to identical protein-level analysis, confirmed by enzyme-linked immunosorbent assay (ELISA) (Figure 6B, D, and F). The amount of protein for ELISA analysis was quantified by analyzing the absorption spectrum using a standard curve (Figure S6). Furthermore, covalent conjugation of MTX to nanotubes significantly inhibited TNF- $\alpha$, IL-1 $\beta$, and IL-6 in inflammatory FLS at the protein level and confirmed by ELISA analysis. In contrast, non-covalent conjugation of MTX on nanotubes showed comparatively low anti-inflammatory ability in inflammatory FLSs (Figure 6B, D, and F). This has already been predicted by uptake analysis, drug release, and intracellular trafficking analysis in this study (Figures 3-5). Based on all obtained results, covalent conjugation of MTX on nanotubes possessed the greatest antiinflammatory ability compared to non-covalent conjugation of MTX on nanotubes and was thus highly effective even at low concentrations of MTX.

\section{Conclusion}

The structural analysis of nanodrugs is a very important factor in understanding and elucidating nanodrug delivery systems. This is because the nanodrug efficacy significantly depends on the structural variation of the nanotubeattached drugs (ie, conjugation styles).$^{30}$ Previous studies have clearly shown that the interaction between nanomaterials and plasma proteins and alterations in the structure of proteins can affect subsequent immune cell functions. ${ }^{41-43}$ These results indicate that structural changes of attached proteins or drugs on nanoparticles can increase drug efficacy through optimal drug regulation in intracellular delivery systems. ${ }^{3,42,43}$ Unfortunately, there have been insufficient studies on the differences in the structural behavior of nanoparticles and drugs according to the binding methods and the associated differences in intracellular drug delivery. In this study, structural changes of conjugated anti-inflammatory drugs (MTX) and nanotubes were compared in the widely used manner (ie, covalent and non-covalent conjugation), and their anti-inflammatory effects were demonstrated in inflammatory synovial cells. In previous studies, non-covalent conjugation of dexamethasone (DEX) to CNTs was very effective in inhibiting FLS-induced inflammation in RA. ${ }^{44}$ However, the most effective drug delivery system requires profound understanding of structural analysis at the atomic level of drug conformation on nanoprobes (NMR analysis). Without analyzing the conformational variation of the attached drugs surrounding nanoparticles, an understanding of structure-associated drug efficacy would be limited. Our study showed that the structural difference of MTX surrounding nanotubes clearly influences drug efficacy with optimal intracellular drug delivery. Interestingly, a huge difference in drug efficacy originated from conjugation styles on nanotubes, as identified in this study. Specifically, the orientation between rings $\mathrm{AB}$ and $\mathrm{C}$ in MTX structures and the conformation of the terminal carboxylic groups are largely affected by covalent conjugation. This indicates that covalent conjugation of MTX on nanotubes induces more structural distortion than the non-covalent conjugation of MTX on nanotubes. Therefore, this study demonstrates that understanding the structural variation of the drug at the atomic level is key to anticipating the subsequent drug efficacy and can provide useful guidelines for designing optimal nanodrug conjugation styles in future nanodrug development systems.

\section{Acknowledgments}

This research was also supported by the National Research Foundation of Korea (NRF) funded by the Ministry of Education, Science, and Technology (2019M3A9H 1103690, 2021R1A5A2030333 and 2020R1A6A1A0304370812). This work was also supported by the Center for Women In Science, Engineering and Technology (WISET) Grant funded by the Ministry of Science and ICT under the Program for Returners into R\&D. We thank beamline $11 \mathrm{C}$ staff at Pohang Accelerator Laboratory (PAL, Korea).

\section{Disclosure}

The authors report no conflicts of interest in this work. 


\section{References}

1. Donahue ND, Acar H, Wilhelm S. Concepts of nanoparticle cellular uptake, intracellular trafficking, and kinetics in nanomedicine. $A d v$ Drug Deliv Rev. 2019;143:68-96.

2. De Volder MF, Tawfick SH, Baughman RH, et al. Carbon nanotubes: present and future commercial applications. Science. 2013;339 (6119):535-539. doi:10.1126/science.1222453

3. Liu Y, Yang Y, Zhang Q, et al. Dynamics of delivering aptamer targeted nano-drugs into cells. J Mater Chem B. 2021;9 (4):952-957. doi:10.1039/D0TB02527E

4. Chakrabarti M, Robinson JT, Tabakman SM, et al. Carbon nanomaterials for drug delivery and cancer therapy. J Nanosci Nanotechnol. 2015;15(8):5501-5511. doi:10.1166/jnn.2015.10614

5. Hao BJ, Li W, Zhang S, et al. A facile PEG/thiol-functionalized nanographene oxide carrier with an appropriate glutathione-responsive switch. Polym Chem. 2020;11(16):2923. doi:10.1039/D0PY90048F

6. Tran TTD, Tran PHL. Nanoconjugation and encapsulation strategies for improving drug delivery and therapeutic efficacy of poorly water-soluble drugs. Pharmaceutics. 2019;11(7):325. doi:10.3390/ pharmaceutics 11070325

7. Liu Z, Tabakman SM, Chen Z, et al. Preparation of carbon nanotube bioconjugates for biomedical applications. Nat Protoc. 2009;4 (9):1372-1382. doi:10.1038/nprot.2009.146

8. Yadav S, Sharma AK, Kumar P. Nanoscale self-assembly for therapeutic delivery. Front Bioeng Biotechnol. 2020;8:127. doi:10.3389/ fbioe.2020.00127

9. Feng C, Huang XY. Polymer brushes: efficient synthesis and applications. Acc Chem Res. 2018;51(9):2314-2323. doi:10.1021/ acs.accounts.8b00307

10. Que YR, Liu Y, Tan W, et al. Enhancing photodynamic therapy efficacy by using fluorinated nanoplatform. ACS Macro Lett. 2016;5 (2):168-173. doi:10.1021/acsmacrolett.5b00935

11. Li RR, Huang X, Lu G, et al. Sulfur dioxide signaling molecule-responsive polymeric nanoparticles. Biomateri Sci. 2020;8 (8):2300-2307. doi:10.1039/D0BM00276C

12. Rajora AK, Ravishankar D, Zhang H, et al. Recent advances and impact of chemotherapeutic and antiangiogenic nanoformulations for combination cancer therapy. Pharmaceutics. 2020;12(6):592. doi:10.3390/pharmaceutics 12060592

13. Park SJ. Protein-nanoparticle interaction: corona formation and conformational changes in proteins on nanoparticles. Int J Nanomedicine. 2020;15:5783-5802. doi:10.2147/IJN.S254808

14. Skubisz MM, Tong S. The evolution of methotrexate as a treatment for ectopic pregnancy and gestational trophoblastic neoplasia: a review. ISRN Obstet Gynecol. 2012;2012:637094. doi:10.5402/2012/637094

15. Fawal MA, Jungas T, Davy A. Inhibition of DHFR targets the self-renewing potential of brain tumor initiating cells. Cancer Lett. 2021;503:129-137. doi:10.1016/j.canlet.2021.01.026

16. Chai D, Hao B, Hu R, et al. Delivery of oridonin and methotrexate via pegylated graphene oxide. ACS Appl Mater Interfaces. 2019;11 (26):22915-22924. doi:10.1021/acsami.9b03983

17. Lopez-Rodriguez R, Ferreiro-Iglesias A, Lima A, et al. Evaluation of a clinical pharmacogenetics model to predict methotrexate response in patients with rheumatoid arthritis. Pharmacogenomics $J$. 2018;18 (4):539-545. doi:10.1038/s41397-018-0017-5

18. Lucas CJ, Dimmitt SB, Martin JH. Optimising low-dose methotrexate for rheumatoid arthritis-A review. Br J Clin Pharmacol. 2019;85 (10):2228-2234. doi:10.1111/bcp.14057

19. Braun J. Methotrexate: optimizing the efficacy in rheumatoid arthritis. Ther Adv Musculoskelet Dis. 2011;3(3):151-158. doi:10.11 77/1759720X11408635

20. Choy E, Aletaha D, Behrens F,et al. Monotherapy with biologic disease-modifying anti-rheumatic drugs in rheumatoid arthritis. Rheumatology (Oxford). 2017;56(5):689-697.
21. Wang G, Peng X. A review of clinical applications and side effects of methotrexate in ophthalmology. J Ophthalmol. 2020;2020:1537689. doi: $10.1155 / 2020 / 1537689$

22. Abbasi M, Mousavi MJ, Jamalzehi S, et al. Strategies toward rheumatoid arthritis therapy; the old and the new. J Cell Physiol. 2019;234(7):10018-10031. doi:10.1002/jcp.27860

23. Chen X, Aletaha D, Behrens F, et al. Targeted hexagonal Pd nanosheet combination therapy for rheumatoid arthritis via the photothermal controlled release of MTX. J Mater Chem B. 2019;7 (1):112-122. doi:10.1039/C8TB02302F

24. Chenthamara D, Subramaniam S, Ramakrishnan SG, et al. Therapeutic efficacy of nanoparticles and routes of administration. Biomater Res. 2019;23:20.

25. Sheikhpour M, Naghinejad M, Kasaeian A, et al. The applications of carbon nanotubes in the diagnosis and treatment of lung cancer: a critical Review. Int $J$ Nanomedicine. 2020;15:7063-7078. doi: $10.2147 /$ IJN.S263238

26. Venkataraman A, Amadi EV, Chen Y, et al. Carbon nanotube assembly and integration for applications. Nanoscale Res Lett. 2019;14(1):220.

27. Cirillo G, Vittorio O, Kunhardt D, et al. Combining carbon nanotubes and chitosan for the vectorization of methotrexate to lung cancer cells. Materials (Basel). 2019;12(18):2889. doi:10.3390/ma12182889

28. Kofoed Andersen C, Khatri S, Hansen J, et al. Carbon nanotubes-potent carriers for targeted drug delivery in rheumatoid arthritis. Pharmaceutics. 2021;13(4):453. doi:10.3390/pharmaceutics13040453

29. Muthukumar T, Prabhavathi S, Chamundeeswari M, et al. Biomodified carbon nanoparticles loaded with methotrexate possible carrier for anticancer drug delivery. Mater Sci Eng C Mater Biol Appl. 2014;36:14-19. doi:10.1016/j.msec.2013.11.046

30. Kim SW, Lee YK, Kim S-H, et al. Covalent, non-covalent, encapsulated nanodrug regulate the fate of intra- and extracellular trafficking: impact on cancer and normal cells. Sci Rep. 2017;7(1):6454. doi:10.1038/s41598-017-06796-7

31. Schulte J. Sequential inversion recovery with RIDE-simultaneous suppression of two solvent signals in 170 NMR spectroscopy. J Magn Reson. 1998;134(1):168-170. doi:10.1006/jmre.1998.1519

32. Piotto M, Saudek V, Sklenar V. Gradient-tailored excitation for single-quantum NMR spectroscopy of aqueous solutions. $J$ Biomol NMR. 1992;2(6):661-665. doi:10.1007/BF02192855

33. Broeren MA, de Waal BFM, van Genderen MHP, et al. Multicomponent host-guest chemistry of carboxylic acid and phosphonic acid based guests with dendritic hosts: an NMR study. $J$ Am Chem Soc. 2005;127(29):10334-10343. doi:10.1021/ja052074m

34. Case DA, Betz RM, Cerutti DS, et al. AMBER 2015. San Francisco: University of California; 2015.

35. Cheng Y, Li Y, Wu Q, et al. Generation-dependent encapsulation/ electrostatic attachment of phenobarbital molecules by poly(amidoamine) dendrimers: evidence from 2D-NOESY investigations. Eur J Med Chem. 2009;44(5):2219-2223. doi:10.1016/j.ejmech.2008.05.031

36. Nam EJ, Kang JH, Sung S, et al. A matrix metalloproteinase 1-cleavable composite peptide derived from transforming growth factor beta-inducible gene h3 potently inhibits collagen-induced arthritis. Arthritis Rheum. 2013;65(7):1753-1763. doi:10.1002/art.37932

37. Lee DU, Park J-Y, Kwon S, et al. Apoptotic lysosomal proton sponge effect in tumor tissue by cationic gold nanorods. Nanoscale. 2019;11 (42):19980-19993. doi:10.1039/C9NR04323C

38. Aswad H, Jalabert A, Rome S. Depleting extracellular vesicles from fetal bovine serum alters proliferation and differentiation of skeletal muscle cells in vitro. BMC Biotechnol. 2016;16(1):32. doi:10.1186/ s12896-016-0262-0

39. Affleck JG, Nowickyj SM, Walker VK. Selection for methotrexate resistance in mammalian cells bearing a Drosophila dihydrofolate reductase transgene: methotrexate resistance in transgenic mammalian cells. Cell Biol Toxicol. 2010;26(2):117-126. doi:10.1007/ s10565-009-9122-1 
40. Lee YK, Choi J, Wang W, et al. Nullifying tumor efflux by prolonged endolysosome vesicles: development of low dose anticancer-carbon nanotube drug. ACS Nano. 2013;7(10):8484-8497. doi:10.1021/ nn4041206

41. Saptarshi SR, Duschl A, Lopata AL. Interaction of nanoparticles with proteins: relation to bio-reactivity of the nanoparticle. J Nanobiotechnology. 2013;11(1):26. doi:10.1186/1477-3155-11-26

42. Francia V, Yang K, Deville S, et al. Corona composition can affect the mechanisms cells use to internalize nanoparticles. ACS Nano. 2019;13(10):11107-11121. doi:10.1021/acsnano.9b03824
43. Park J-Y, Park SJ, Park JY, et al. Unfolded protein corona surrounding nanotubes influence the innate and adaptive immune system. Adv Sci (Weinh). 2021;8(8):2004979. doi:10.1002/ advs.202004979

44. Lee YK, Kim SW, Park JY, et al. Suppression of human arthritis synovial fibroblasts inflammation using dexamethasone-carbon nanotubes via increasing caveolin-dependent endocytosis and recovering mitochondrial membrane potential. Int $J$ Nanomedicine. 2017;12:5761-5779. doi:10.2147/IJN.S142122

\section{Publish your work in this journal}

The International Journal of Nanomedicine is an international, peerreviewed journal focusing on the application of nanotechnology in diagnostics, therapeutics, and drug delivery systems throughout the biomedical field. This journal is indexed on PubMed Central, MedLine, CAS, SciSearch ${ }^{\mathbb{R}}$, Current Contents ${ }^{\mathbb{R}} /$ Clinical Medicine,
Journal Citation Reports/Science Edition, EMBase, Scopus and the Elsevier Bibliographic databases. The manuscript management system is completely online and includes a very quick and fair peer-review system, which is all easy to use. Visit http://www.dovepress.com/ testimonials.php to read real quotes from published authors. 